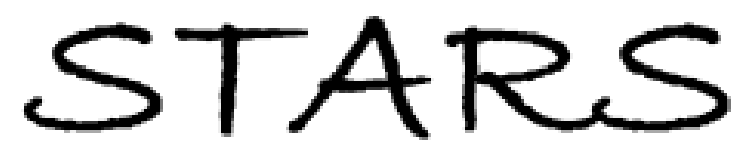

University of Central Florida

STARS

$1-1-2011$

\title{
Thermodynamics and kinetics of silicon under conditions of strong electronic excitation
}

\author{
Lalit Shokeen \\ University of Central Florida \\ Patrick K. Schelling \\ University of Central Florida
}

Find similar works at: https://stars.library.ucf.edu/facultybib2010 University of Central Florida Libraries http://library.ucf.edu

This Article is brought to you for free and open access by the Faculty Bibliography at STARS. It has been accepted for inclusion in Faculty Bibliography 2010 s by an authorized administrator of STARS. For more information, please contact STARS@ucf.edu.

\section{Recommended Citation}

Shokeen, Lalit and Schelling, Patrick K., "Thermodynamics and kinetics of silicon under conditions of strong electronic excitation" (2011). Faculty Bibliography 2010s. 1915.

https://stars.library.ucf.edu/facultybib2010/1915

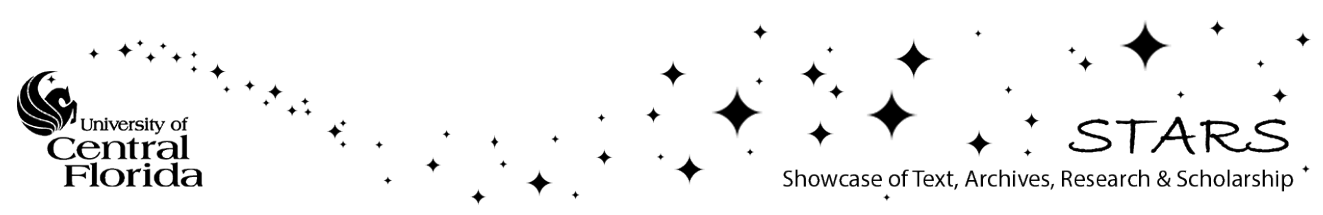




\section{Thermodynamics and kinetics of silicon under conditions of strong electronic excitation}

Cite as: J. Appl. Phys. 109, 073503 (2011); https://doi.org/10.1063/1.3554410

Submitted: 28 October 2010 . Accepted: 11 January 2011 . Published Online: 01 April 2011

Lalit Shokeen, and Patrick K. Schelling

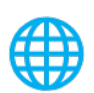

\section{ARTICLES YOU MAY BE INTERESTED IN}

An empirical potential for silicon under conditions of strong electronic excitation

Applied Physics Letters 97, 151907 (2010); https://doi.org/10.1063/1.3499296

Atomic-scale simulation of the thermodiffusion of hydrogen in palladium

Journal of Applied Physics 114, 063509 (2013); https://doi.org/10.1063/1.4816961

Molecular-dynamics calculation of the vacancy heat of transport

Journal of Applied Physics 116, 023506 (2014); https://doi.org/10.1063/1.4886577

\section{Applied Physics Reviews} Now accepting original research

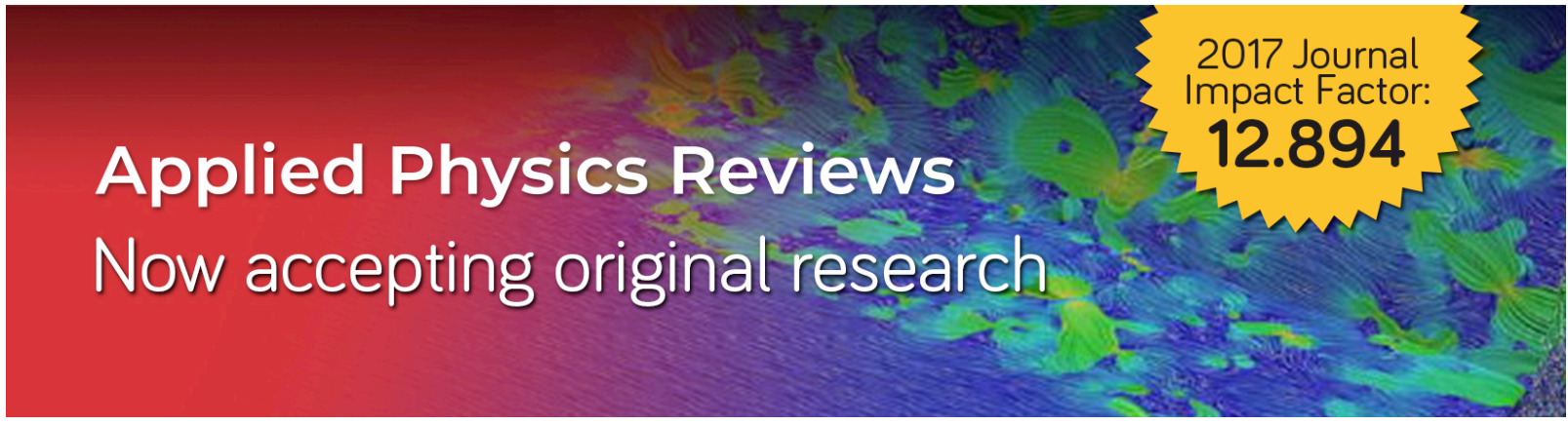




\title{
Thermodynamics and kinetics of silicon under conditions of strong electronic excitation
}

\author{
Lalit Shokeen and Patrick K. Schelling ${ }^{\text {a) }}$ \\ Advanced Material Processing and Analysis Center and Department of Physics University of Central Florida, \\ 4000 Central Florida Blvd., Orlando, Florida 32816, USA
}

(Received 28 October 2010; accepted 11 January 2011; published online 1 April 2011)

\begin{abstract}
We present a detailed analysis of a recently-developed empirical potential to describe silicon under conditions of strong electronic excitation. The parameters of the potential are given as smooth functions of the electronic temperature $T_{e}$, with the dependence determined by fitting to finite-temperature density-functional theory calculations. We analyze the thermodynamics of this potential as a function of the electronic temperature $T_{e}$ and lattice temperature $T_{i o n}$. The potential predicts phonon spectra in good agreement with finite-temperature density-functional theory, including the previously predicted lattice instability. We predict that the melting temperature $T_{m}$ decreases strongly as a function of $T_{e}$. Electronic excitation has a strong effect on the rate of crystallization from the melt. In particular, high $T_{e}$ results in very slow kinetics for growing crystal from the melt, due mainly to the fact that diamond becomes much less stable as $T_{e}$ increases. Finally, we explore annealing amorphous $\mathrm{Si}$ (a-Si) below $T_{m}$, and find that we cannot observe annealing of a-Si directly at high $T_{e}$. We hypothesize that this is also due to the decreased stability of the diamond structure at high $T_{e}$. (ㅇ 2011 American Institute of Physics. [doi:10.1063/1.3554410]
\end{abstract}

\section{INTRODUCTION}

The ability to generate short, intense laser pulses has opened many possible avenues for materials processing. One particular advantage is the ability to process or even ablate a surface, while maintaining an overall low lattice temperature. This is a definite advantage, for example, when processing a silicon wafer that includes integrated materials with a melting temperature below that of silicon. This is possible because, for ultra-short (100 fs or less) laser pulses, the bonds can be destabilized, leading to a direct athermal transition to a liquid state in very short time (less than $100 \mathrm{fs}$ ). This occurs because high densities of electron-hole pairs are generated which destabilizes the lattice. For example, during a $100 \mathrm{fs}$ laser pulse of wavelength $\lambda=625 \mathrm{~nm}$, it has been predicted that the temperature of the electron-hole plasma can attain extremely high temperatures, with $k_{B} T_{e}$ at least $1 \mathrm{eV}$ when the fluence is at least $100 \mathrm{~mJ} / \mathrm{cm}^{2}{ }^{1}$. The electronhole density can reach $\sim 10^{22} \mathrm{~cm}^{-3}$ under these conditions. The production of liquid by an athermal transition has been verified by several groups. For example, time-resolved reflectivity measurements have been used to track melting in optically-excited silicon. ${ }^{2}$ More recently, in situ x-ray diffraction has provided direct visualization of the atomic motion in indium antimonide. ${ }^{3}$

There is a definite lack of theoretical tools available to understand materials processing under these conditions of extreme electronic excitation. At the most fundamental level, several insightful studies have been performed using either finite-temperature density-functional theory (DFT) $)^{4-6}$ or tightbinding. ${ }^{7}$ However, to access the long length and time scales needed to elucidate questions related to materials processing,

${ }^{\text {a)} E l e c t r o n i c ~ m a i l: ~ p s c h e l l @ m a i l . u c f . e d u . ~}$ molecular-dynamics (MD) simulation with empirical potentials is the standard approach. ${ }^{8}$ While there are many useful MD studies of laser processing, it is important to note that previous MD simulations have ignored the physics of lattice instabilities caused by electronic excitation.

Only recently has there been any effort to develop empirical potentials that can treat materials in a highly-excited electronic state. Recently an empirical potential for tungsten has been developed and parameterized using finite-temperature DFT calculations. ${ }^{9}$ In a recent article, we have presented a parameterization for an empirical potential that describes silicon under conditions of strong electronic excitation. ${ }^{10}$ The potential was parameterized by fitting directly to finite-temperature DFT calculations. We have reported the parameterization for only four excitation conditions. The empirical potential was used to simulate athermal melting, and reasonable agreement with finite-temperature DFT studies was demonstrated. ${ }^{10}$ In particular, we found that strong excitation leads to fast ( $\sim 100 \mathrm{fs})$, athermal melting of the lattice with a strong increase in ion temperature. Interestingly, we also demonstrated that somewhat below the conditions needed for destabilization of the diamond lattice, cooling occurs at least for very short times. This effect was due to the fact that electronic excitation initially weakens the bonds between the atoms and decreases the restoring forces. We were also able to show that when the electronic system is strongly excited, an ordinary first-order melting transition might still occur, but at greatly reduced melting temperatures.

In this article, we extend the previous study. ${ }^{10}$ We present a parameterization of the potential that can be applied across a range of excitations from $k_{B} T_{e}=0$ to $k_{B} T_{e}=2.50$ $\mathrm{eV}$. To achieve this, we find sixth-order polynomials that describe the dependence of the parameters on $k_{B} T_{e}$. We apply the method to elucidate ordinary thermal melting that occurs under conditions of strong electronic excitation. In 
contrast to athermal melting, we predict that thermal melting will occur over longer timescales but should be apparent at dramatically reduced melting temperature $T_{m}$. We also characterize the kinetics of the liquid-solid transition, transport properties of the liquid, and annealing of amorphous silicon.

\section{APPROACH}

As described in Ref. 10, the database used to develop the empirical potentials was obtained from finite-temperature DFT calculations using the ABINIT code. ${ }^{11}$ The conditions for our calculations were quite close to that given in Ref. 6 and are presented in Ref. 10. Overall, as $k_{B} T_{e}$ increases, finite-temperature DFT predicts that the stability of the diamond phase decreases with respect to the other phases. In addition, we find that the equilibrium lattice parameter for diamond tends to increase with $k_{B} T_{e}$. These observations are consistent with the notion that a strong laser pulse will tend to destabilize the diamond phase and may induce a transition to a phase with higher atomic coordination, including the possibility of an athermal transition to the liquid state.

The empirical potential is based on a recently-published modified Tersoff formalism (called the MOD potential in Ref. 12), which has been shown to provide an excellent description of the phase behavior and kinetics. ${ }^{12,13}$ The total energy $\Phi$ is determined from the terms,

$$
\begin{gathered}
\Phi=\frac{1}{2} \sum_{i \neq j} \phi_{i j}\left(r_{i j}\right), \\
\phi_{i j}\left(r_{i j}\right)=f_{c}\left(r_{i j}\right)\left[A \exp \left(-\lambda_{1} r_{i j}\right)-b_{i j} B \exp \left(-\lambda_{2} r_{i j}\right)\right], \\
b_{i j}=\left(1+\xi_{i j}^{\eta}\right)^{-\delta}, \\
\xi_{i j}=\sum_{k \neq i, j} f_{c}\left(r_{i j}\right) g\left(\theta_{i j k}\right) \exp \left[\alpha\left(r_{i j}-r_{i k}\right)^{\beta}\right],
\end{gathered}
$$

where $r_{i j}$ is the interatomic separation and $\theta_{i j k}$ is the bond angle between $i j$ and $i k$. The function $\mathrm{g}(\theta)$ has been modified from the Tersoff model, and is given for the MOD potential by

$$
\begin{gathered}
g(\theta)=c_{1}+g_{o}(\theta) g_{a}(\theta), \\
g_{o}(\theta)=\frac{c_{2}(h-\cos \theta)^{2}}{c_{3}+(h-\cos \theta)^{2}},
\end{gathered}
$$

$$
g_{a}(\theta)=1+c_{4} \exp \left[-c_{5}(h-\cos \theta)^{2}\right]
$$

The cutoff function $f_{c}(r)$ has been modified from the original Tersoff potential. For $r \leq R_{1}, f_{c}(r)=1$, and for $r \geq R_{2}=0$, $f_{c}(r)=0$. In the region $R_{1}<r<R_{2}$, the cutoff function is

$$
f_{c}(r)=\frac{1}{2}+\frac{9}{16} \cos \left(\pi \frac{r-R_{1}}{R_{2}-R_{1}}\right)-\frac{1}{16} \cos \left(3 \pi \frac{r-R_{1}}{R_{2}-R_{1}}\right) .
$$

For $k_{B} T_{e}=0$, we use the original formulation except with larger cutoffs $\left(R_{1}=3.1 \AA\right.$ and $\left.R_{2}=3.4 \AA\right)$ and $\alpha=1.90$ chosen to give a melting temperature $T_{m}=1688^{\circ} \mathrm{K}$ close to experiment. For finite $k_{B} T_{e}$, the parameters were fit to reproduce the DFT cohesive energies for the fcc, bcc, simple cubic, and diamond structures at seven different volumes. Some parameters were found to not be critical, and hence were held fixed. Because the potential for $k_{B} T_{e}=0$ was not fit to the DFT, we fit instead to changes in the free energy curves as a function of $k_{B} T_{e}$. Also, the DFT energies were shifted to give the experimental value $E_{c o h}=4.63 \mathrm{eV}$ in the equilibrium structure.

Because the potential was fit in intervals of $0.05 \mathrm{eV}$, it was possible to obtain a smooth dependency on $k_{B} T_{e}$. After fitting the parameters for each $k_{B} T_{e}$, we then fit the $k_{B} T_{e}$ dependence to a polynomial of order 6 . For example, the parameter $A\left(T_{e}\right)$, which is the strength of the repulsive interaction (in $\mathrm{eV}$ ), depends on $T_{e}$ in the form

$$
A\left(T_{e}\right)=\sum_{n=0}^{6} a_{n}\left(k_{B} T_{e}\right)^{n}
$$

In Table I, we show the coefficients of the parameterization. For the parameters $a_{0}$ in each case, we use the parameters from the original modified Tersoff potential. ${ }^{12}$ The only exception is the parameter $\alpha$ which, as noted already, we take to be $\alpha=1.90$ at $k_{B} T_{e}=0$. We include in Table I a constant $F_{0}$ that is added to the empirical potential to take into

\begin{tabular}{|c|c|c|c|c|c|c|c|}
\hline$n$ & $a_{0}$ & $a_{1}$ & $a_{2}$ & $a_{3}$ & $a_{4}$ & $a_{5}$ & $a_{6}$ \\
\hline$A(e V)$ & 3281.5905 & 6.709 & -2228.600 & 2188.300 & -1079.800 & 297.010 & -35.320 \\
\hline$B(e V)$ & 121.00047 & -15.679 & -171.110 & 237.280 & -145.080 & 42.934 & -4.972 \\
\hline$\lambda_{2}\left(\AA^{-1}\right)$ & 1.3457970 & 0.0444 & -0.7608 & 0.4223 & -0.1344 & 0.0363 & -0.0055 \\
\hline$\eta \times \delta$ & 0.53298909 & -0.0462 & 0.6076 & -0.4737 & 0.1070 & 0.0128 & -0.0055 \\
\hline$\alpha$ & 1.9000 & -0.3901 & -0.1281 & 0.0291 & 0.0735 & -0.4140 & 0.0066 \\
\hline$c_{1}$ & 0.20173476 & -0.2709 & 0.8593 & -0.8428 & 0.7992 & -0.3732 & 0.0600 \\
\hline$c_{2}$ & 730418.72 & -100721.0 & -808138.0 & 503890.0 & -16238.0 & -40502.0 & 5940.3 \\
\hline$c_{4}$ & 1.0 & 0.0180 & 0.0 & 0.0 & 0.0 & 0.0 & 0.0 \\
\hline$F_{0}(e V)$ & 0 & -0.0290 & 0.2325 & -0.6229 & 0.6668 & -0.2421 & 0.0299 \\
\hline
\end{tabular}
account the fact that the free energy of an isolated silicon atom will also depend on $k_{B} T_{e}$.

In the previous paper reporting the potential, the dependence of the lattice parameter and bulk modulus on $k_{B} T_{e}$ was shown to be in good agreement with finite-temperature

TABLE I. Expansion coefficients for the power-series representation of the dependence of the potential parameters on $k_{B} T_{e}$. The notation follows Ref. 12 . The physical dimensions of the expansion coefficients are not given. However, the dimensions of the potential parameters are given in the left column, and the dimensions of the expansion coefficients can be inferred. 
DFT results. ${ }^{10}$ Here, in Fig. 1 we present the cohesive energy per atom $\Delta F_{c o h}$ of the various phases relative to the diamond phase as a function of $k_{B} T_{e}$ at the atomic volume $\Omega_{a t}=20.0 \AA^{3}$. It is evident that, for $k_{B} T_{e}=0$, the diamond phase has a much larger cohesive energy $\left(\Delta F_{c o h}<0\right.$ for each phase) than the other phases as expected. The empirical potential at $k_{B} T_{e}=0$, which is nearly identical to the MOD potential, overestimates the difference between diamond and the other phases. Because we fit the changes with respect to $k_{B} T_{e}$, rather than to absolute values of $\Delta F_{c o h}$, the general dependence on $k_{B} T_{e}$ is well reproduced. In particular, as $k_{B} T_{e}$ increases, the cohesive free energy of the fcc, bcc, and simple cubic phases each approach that of the diamond phase. For the empirical potential, the simple-cubic phase becomes energetically favorable for $\Omega_{a t}=20.0 \AA^{3}\left(\Delta F_{c o h}>0\right)$ at about $k_{B} T_{e}=1.75 \mathrm{eV}$. By contrast, the finite-temperature DFT calculations predict that the simple-cubic phase becomes favorable at about $k_{B} T_{e}=1.25 \mathrm{eV}$. This difference is due to the initial disagreement in the MOD potential at $k_{B} T_{e}=0$ for the relative energies of the phases. Finally, as $k_{B} T_{e}=2.5 \mathrm{eV}$ is approached, differences between the different crystal structures becomes fairly small, consistent with predictions of finite-temperature DFT. From the $\Delta F_{c o h}$ curves alone, it is possible to understand aspects of the response of silicon to laser excitation. In particular, as the excitation increases, the diamond phase is destabilized with respect to the other crystalline phases. The tendency is for the open diamond structure, which requires strong directional bonding, to become less favorable with respect to higher-coordinated phases. This trend includes the liquid phase which is characterized by coordination numbers larger than 4 . For the liquid phase, we have previously found coordination numbers in excess of those in ordinary liquid silicon. ${ }^{10}$

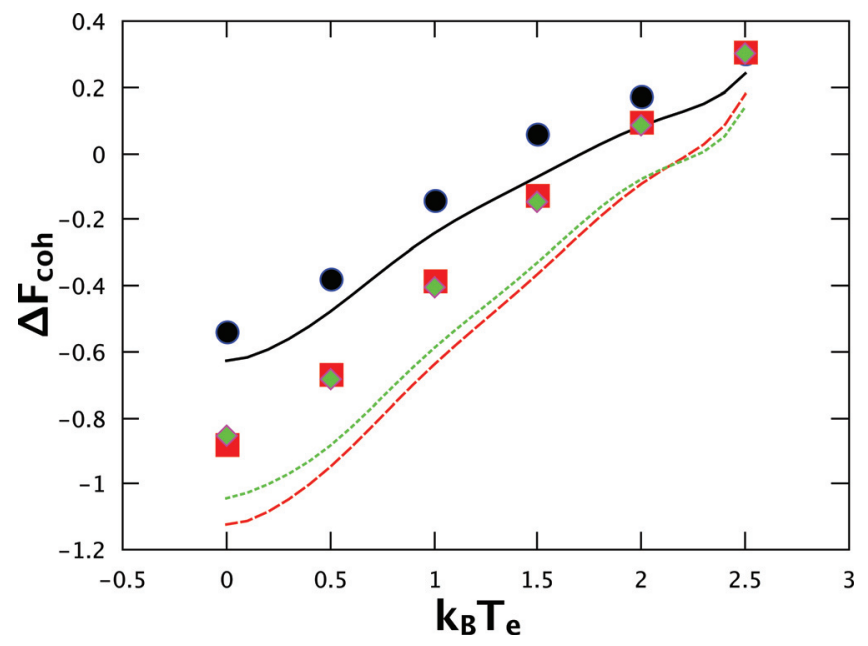

FIG. 1. (Color online) Cohesive free energy $\Delta F_{\text {coh }}$ computed with respect to the diamond phase as a function of $k_{B} T_{e}$ for the simple-cubic (solid line), bcc (dashed line), and fcc (dotted line) phases. Each curve is determined at an atomic volume $\Omega_{a t}=20.0 \AA^{3}$. For comparison, the same quantities from DFT calculations are shown at a few values of $k_{B} T_{e}$ for simple-cubic (circles), bcc (squares), and fcc (diamonds) phases. For low $k_{B} T_{e}$, each phase has a negative value for $\Delta F_{c o h}$, indicating the diamond phase is stable. For very large values of $k_{B} T_{e}$, the values of $\Delta F_{c o h}$ become positive, indicating that diamond is no longer the stable phase.
The differences between the $\Delta F_{c o h}$ predicted by the empirical potential and finite-temperature DFT have some important consequences for the response to a laser pulse. Because the system can experience a rapid increase in $k_{B} T_{e}$, the diamond phase is made unstable before the atoms have been able to experience significant displacements. When the system becomes unstable, the ionic temperature $T_{i o n}$ increases dramatically, as shown in Ref. 10 and finite-temperature DFT simulations. However, because the empirical potential predicts a smaller $\Delta F_{c o h}$ (when $\left.\Delta F_{c o h}>0\right)$ than finite-temperature DFT, the increase in $T_{i o n}$ predicted by the empirical potential is somewhat smaller than what is seen in the finite-temperature DFT results. ${ }^{10}$

Insight into the destabilization of the diamond phase can be obtained by computing the dependence of the phonon frequencies on $k_{B} T_{e}$. As first pointed out in Ref. 6, the instability of the diamond lattice produced by laser excitation can be closely identified with softening of the transverse acoustic (TA) phonon branch. For strong enough excitation, the TA branch becomes unstable, and the restoring forces stabilizing the diamond structure vanish, at which point the crystal will transform into another phase. In Fig. 2 we show the computed phonon frequencies as a function of $k_{B} T_{e}$ for the diamond lattice at the atomic volume $\Omega_{a t}=20.0 \AA^{3}$. It is clear from Fig. 2 that the overall agreement with finite-temperature DFT calculations is reasonably good. In fact, the agreement for high $k_{B} T_{e}$ is substantially better than for $k_{B} T_{e}=0$ where the MOD potential is used. For each mode shown in Fig. 2, it is clear that the electronic excitation weakens the interatomic bonds, which is also reflected in the bulk modulus. ${ }^{10}$ Perhaps most importantly, the TA zone-edge mode becomes unstable at about $k_{B} T_{\mathrm{e}}=1.45 \mathrm{eV}$. While the empirical potential yields a TA instability at a somewhat lower temperature than for the finite-temperature DFT case, it demonstrates the right trends and qualitative behavior.

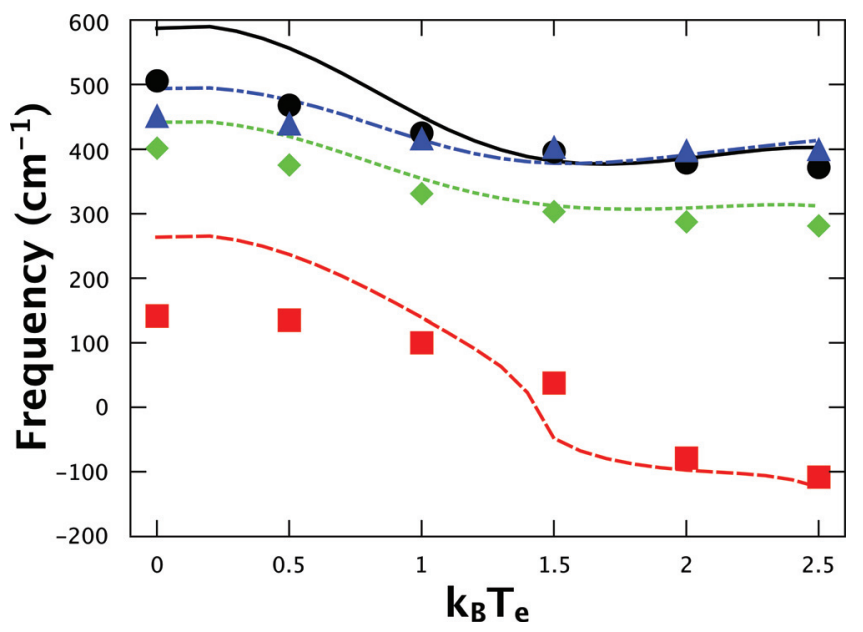

FIG. 2. (Color online) Phonon frequencies computed by the empirical potential for the $\Gamma$-point LO/TO (solid line), and $X$-point LA/LO (dotted line), TO (dot-dashed line), and TA (dashed line) branches in the diamond lattice with $\Omega_{a t}=20.0 \AA^{3}$ as a function of the electronic temperature $k_{B} T_{e}$. The finite-temperature DFT results are also shown for the $\Gamma$-point LO/TO (circles), and $X$-point LA/LO (diamonds), TO (triangles), and TA (squares). Negative frequencies in the TA branch correspond to instabilities. 
In summary, we have presented a parameterization of the empirical potential for silicon, and determined several properties as a function of $k_{B} T_{e}$ at zero lattice temperature $T_{i o n}=0$. The bond-weakening that results in destabilization of the diamond lattice is fairly well described by the empirical potential. For example, the instability of the TA phonon branch and the decrease in the bulk modulus seen for higher values of $k_{B} T_{e}$ is in good agreement with finite-temperature DFT results that were used in the fitting. In the next section, we explore the thermodynamics of the empirical potential for finite values of $T_{i o n}$.

\section{THERMODYNAMICS AND KINETICS FOR FINITE $T_{\text {ion }}$}

When $k_{B} T_{e}$ becomes high, the interatomic bonds are greatly altered due to electron-hole excitations. As a result, the thermodynamic properties will depend on $T_{i o n}$ in a manner that depends quite strongly on $k_{B} T_{e}$. In this section, we address various aspects of the equilibrium thermodynamics and kinetics of the empirical potential. It is conceptually important to recognize that strictly speaking equilibrium thermodynamics is not realized unless $T_{i o n}=T_{e}$. However, within the simulations reported here, it is always possible to fix $T_{i o n}$ and $T_{e}$ independently, since $T_{e}$ only determines the nature of the interatomic potential. Hence, the simulations here represent the real thermodynamic and kinetic properties of the potential. However, it may be very difficult to verify the results experimentally. Nevertheless, it has previously been shown that equilibrium thermodynamic properties can often be relevant to developing an understanding of strong nonequilibrium physics, including in studies of laser ablation of silicon. ${ }^{8}$ We highlight some of the relevant properties of the empirical potential in the subsections below.

\section{A. Lattice thermal expansion}

Constant-pressure calculations of diamond using the Parrinello-Rahman algorithm ${ }^{14}$ were used to determine the zero-pressure lattice parameters as a function of the lattice temperature $T_{i o n}$ and electronic energy $k_{B} T_{e}$. The results are shown in Fig. 3. As previously demonstrated, ${ }^{10}$ increasing $k_{B} T_{e}$ results in weakened interatomic interactions and a substantial increase in the lattice parameter $a$. The dependence of $a$ on $T_{i o n}$ shows interesting behavior as $k_{B} T_{e}$ increases. In particular, for $k_{B} T_{e}$ below $1.0 \mathrm{eV}$, the thermal expansion coefficient $\alpha_{L}$, computed at $T_{i o n}=150^{\circ} \mathrm{K}$, depends only weakly on $k_{B} T_{e}$, as shown in Fig. 4. However, above $k_{B} T_{e}=$ $1.0 \mathrm{eV}, \alpha_{L}$ decreases dramatically and eventually becomes strongly negative, especially at $k_{B} T_{e}=1.50 \mathrm{eV}$. Further increasing $k_{B} T_{e}$ above $1.50 \mathrm{eV}$ results in $\alpha_{L}$ values closer to zero. This shows that, after the initial fast expansion that occurs upon laser excitation, the thermal expansion of the crystal follows rather complex behavior.

\section{B. Melting transition}

The melting temperature $T_{m}$ was determined from simulations of coexistence at zero pressure. ${ }^{13,15,16} \mathrm{We}$ simulate a system that begins with a $6 \times 6 \times 40$ diamond lattice with

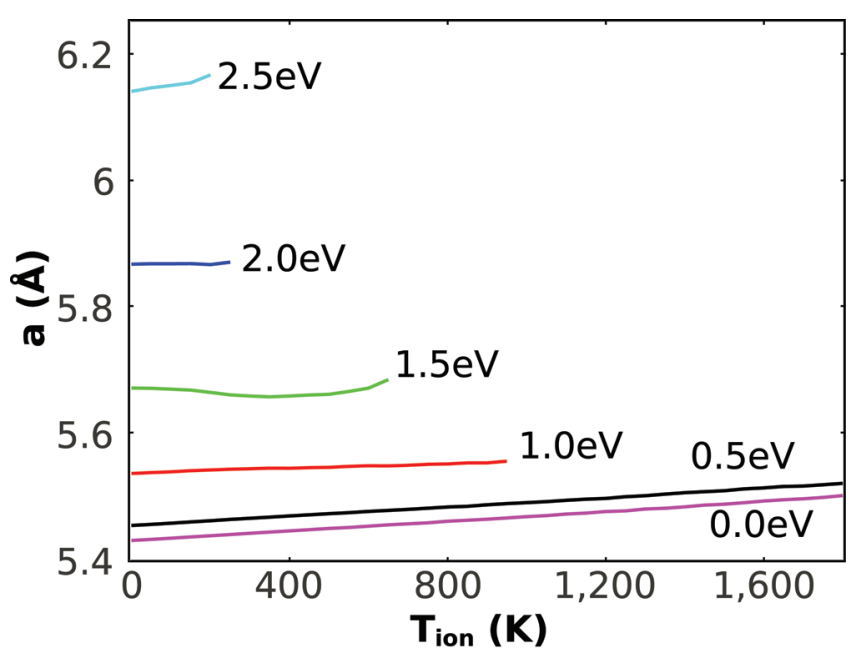

FIG. 3. (Color online) Lattice parameter $a$ of diamond plotted as a function of the lattice temperature $T_{i o n}$ plotted for several values of the electronic temperature $k_{B} T_{e}$. The curves terminate near the point where the crystal transforms into a liquid.

11520 atoms. First, the system is equilibrated at constant temperature at an estimated melting temperature for $5.52 \mathrm{ps}$. After this preliminary step, half of the atoms are fixed in place, while the rest are heated to $3000^{\circ} \mathrm{K}$ for $10.52 \mathrm{ps}$ to initiate melting at constant volume. Next, the system is cooled to the estimated melting temperature and equilibrated for 11.05 ps. In the last step, the fixed atoms are released and the entire system is allowed to evolve in a constant energy simulation. For this last step, the simulation cell length perpendicular to the liquid-solid interface is allowed to vary to establish zero stress. In the plane of the interface, the dimensions are fixed at the predicted zero-stress lattice parameters of the crystal. In a constant energy simulation, due to the latent heat of melting, the system will always evolve toward the coexistence point. To get an accurate calculation of $T_{m}$, we simulate for at least 1 ns to average the system temperature once equilibrium is achieved. However, since the estimated $T_{m}$ might be different from what is finally obtained, and the value of $T_{m}$ depends on maintaining the crystal at the

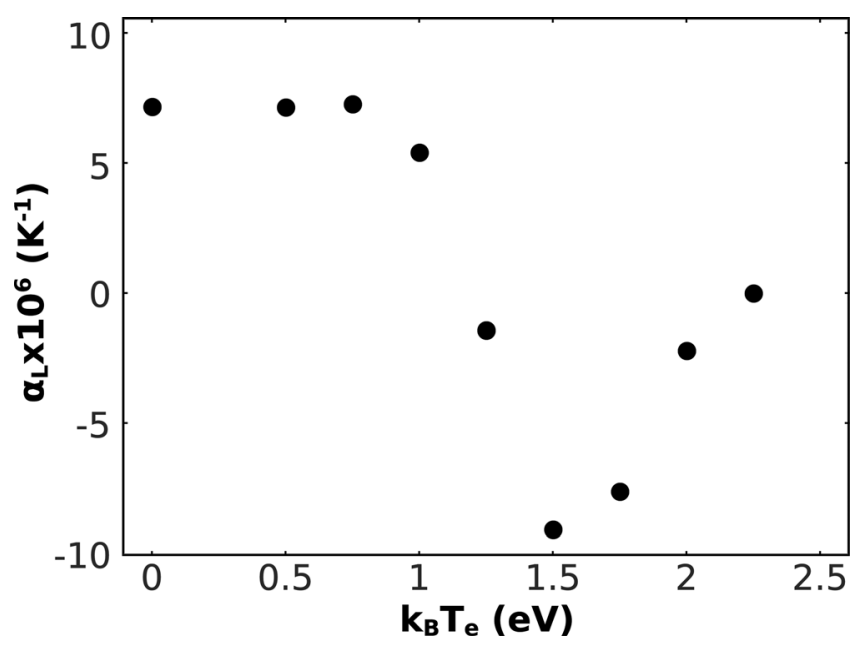

FIG. 4. Thermal expansion coefficient $\alpha$ as a function of $k_{B} T_{e}$ determined at $T_{\text {ion }}=150^{\circ} \mathrm{K}$. 
correct zero-stress condition, the calculation of $T_{m}$ often requires $2-3$ iterations using the results to improve the estimate of $T_{m}$.

The results for $T_{m}$ are shown in Fig. 5. As previously reported, ${ }^{10} T_{m}$ decreases sharply as $k_{B} T_{e}$ increases. ${ }^{10}$ For values of $k_{B} T_{e}$ above about $1.5 \mathrm{eV}$, we were unable to stabilize the diamond phase, which might indicate the presence of some other crystalline phase. Quite significantly, we find that $T_{m} \sim 300^{\circ} \mathrm{K}$ for $k_{B} T_{e} \sim 1.5 \mathrm{eV}$, which indicates clearly that a liquid phase can in principle be realized with only very small increases in the lattice temperature.

\section{Melting kinetics}

We explored the kinetics of the melting transition using constant temperature simulations near the coexistence point. In these calculations, the system was prepared in the same way as the coexistence calculations, but in the final step the system is maintained at constant temperature, resulting in either melting or solidification. In Fig. 6, we see that the rate of melting is quite similar independent of $T_{e}$. By contrast, while the rate crystallization is quite similar for $k_{B} T_{e}=0$ and $k_{B} T_{e}=0.5 \mathrm{eV}$, for the higher temperature case $k_{B} T_{e}=1.25$ $\mathrm{eV}$ crystallization occurs very slowly. The dependence of the kinetics on $k_{B} T_{e}$ arises from two sources. First, the driving force for crystallization is lower for high $k_{B} T_{e}$ due to the fact that the diamond lattice is rendered increasingly unstable. Another factor is the dependence on $k_{B} T_{e}$ of diffusion in the liquid. The kinetics of melting for the MOD potential was previously reported by Schelling. ${ }^{13}$ The present results for the interface velocity with $k_{B} T_{e}=0$ appear to be closer to experimental results than in Ref. 13 for the MOD potential. We include in Fig. 6 the experimental values taken from Refs. 16 and 17. However, in course of analyzing the results reported here, we discovered that the results in Ref. 13 for the MOD potential were unfortunately in error, overestimating the interface velocity by exactly a factor of 2 . However, the details of the potential here for $k_{B} T_{e}=0$ are not exactly equivalent to the MOD potential, in particular having larger cutoffs and a different value for the $\alpha$ parameter, so the

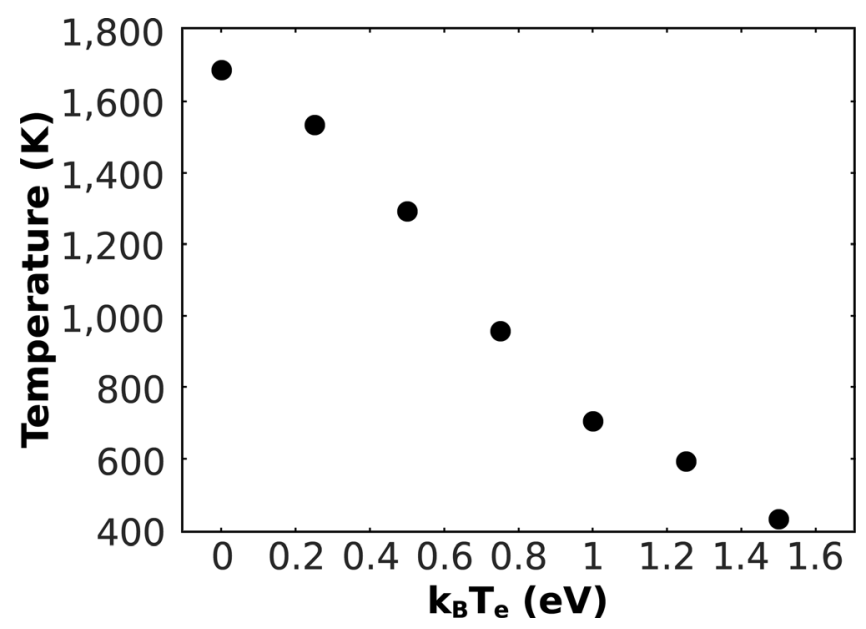

FIG. 5. The melting temperature $T_{m}$ computed using the empirical potential as a function of $k_{B} T_{e}$. Each point was determined at zero pressure from a simulation of the coexistence.

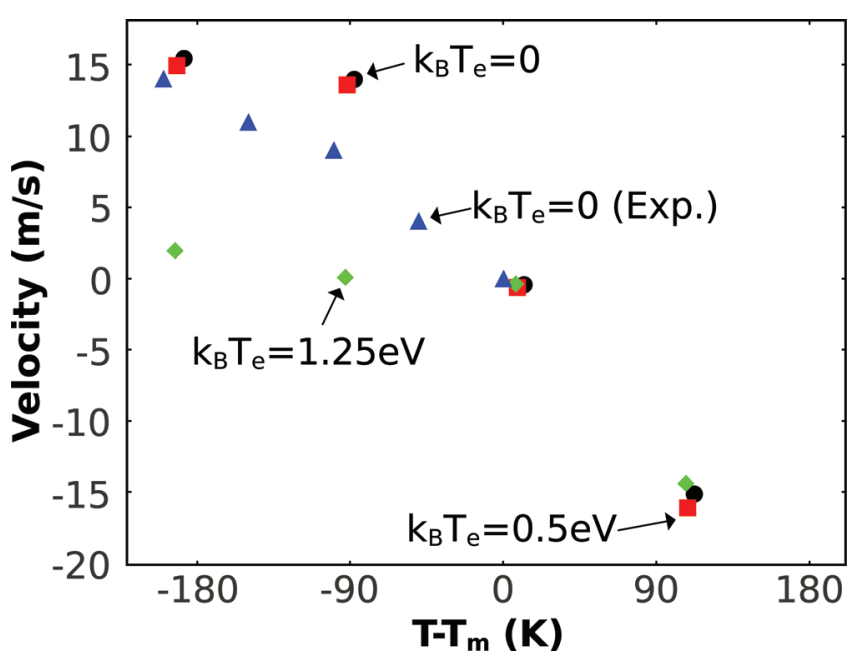

FIG. 6. (Color online) Interface velocity of a liquid-crystal system as a function of $T-T_{m}$. For $T-T_{m}$, crystal growth occurs, and the velocity is positive. Above the melting point, the interface velocity is negative, corresponding to melting. Results for $k_{B} T_{e}=0$ (circles), $k_{B} T_{e}=0.5 \mathrm{eV}$ (squares), and $k_{B} T_{e}=1.25 \mathrm{eV}$ are given. We include experimental results for solidification take from Ref 17, 18 (triangles) which can be compared to the $k_{B} T_{e}=0$ results.

kinetics of the present model at $k_{B} T_{e}=0$ are in fact somewhat different from the MOD potential. We conclude that the kinetics predicted by the present potential for $k_{B} T_{e}=0$ and the MOD potential are somewhat different, but that both in fairly good agreement with experimental measurements of the interface velocity.

\section{Liquid self diffusion}

In the previous article, ${ }^{10}$ we demonstrated that the structure of the liquid was significantly different for high values of $k_{B} T_{e}$ when compared to $T_{e}=0$, in good agreement with finite-temperature DFT results. ${ }^{10}$ Here, we report values of the self-diffusion constant $D$ obtained at the zero-pressure coexistence point for three different values of $k_{B} T_{e}$. We begin with 4096 silicon atoms that are simulated at $3000^{\circ} \mathrm{K}$ for $10.5 \mathrm{ps}$. The system is then cooled to the coexistence point, and statistics for the mean-squared displacement $r_{a v}^{2}$ as a function of time is computed. We show in Fig. $7 r_{a v}^{2}$ as a function of simulation time for $k_{B} T_{e}=0,0.5 \mathrm{eV}$, and 1.25 $\mathrm{eV}$. We use the Einstein relation $r_{a v}^{2}=6 D t$ to determine the diffusion coefficient $D$. In Table II, we show the computed diffusion constants $D$. For $k_{B} T_{e}=0$, we can compare to the experimental value $10^{-4} \mathrm{~cm}^{2} / s .{ }^{19,20}$ From Table II, we see that the MOD potential predicts a value close to experiment and somewhat larger than the Stillinger-Weber potential, ${ }^{21}$ which gives $D=6.94 \times 10^{-5} \mathrm{~cm}^{2} / s .{ }^{19,20}$ As $k_{B} T_{e}$ increases, $D$ actually decreases. This seems counterintuitive at first, since the interatomic interactions are weakened for high $k_{B} T_{e}$. However, since the melting temperature is strongly dependent on $k_{B} T_{e}$, the values of $D$ are obtained at much lower $T_{i o n}$ for high $k_{B} T_{e}$. In short, while we have not yet done an extensive study to obtain the activation energies for diffusion, we do not believe that Fig. 7 is inconsistent with the expectation that the activation energy for self-diffusion in the liquid is less for high $k_{B} T_{e}$. 


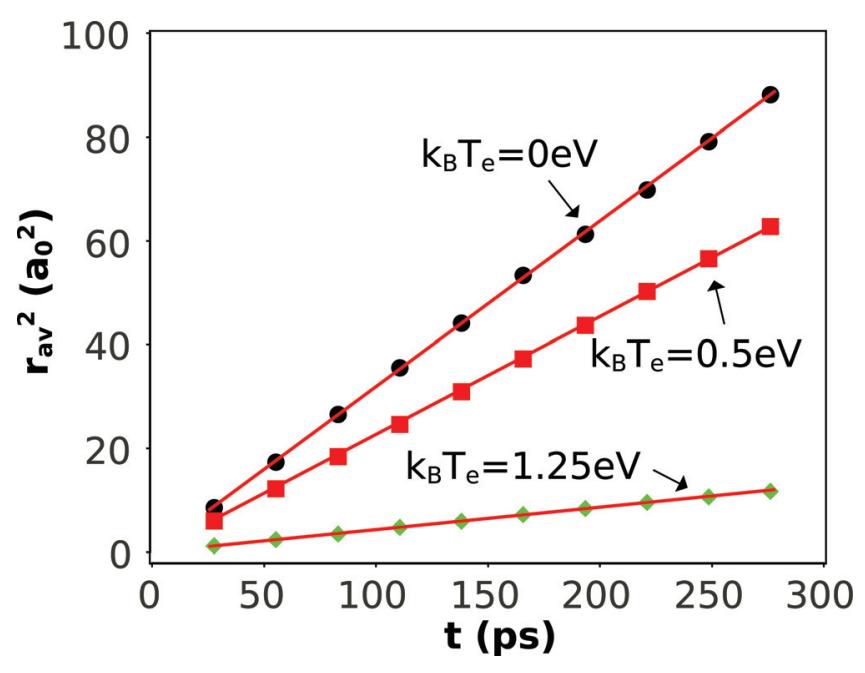

FIG. 7. (Color online) Mean-squared displacement $r_{a v}^{2}$ computed at the coexistence point as a function of simulation time for liquid silicon.

\section{E. Annealing amorphous Si}

We explored the potential for annealing at an interface of amorphous silicon (a-Si) and crystalline silicon by generating strong electronic excitation. This technique is of interest in producing high-quality polycrystalline films from a-Si, primarily by melting the a-Si followed by crystal growth. ${ }^{22}$ However, we explored the potential for direct annealing of a$\mathrm{Si}$ without creation of a liquid. The possibility of forming crystalline silicon directly from a-Si without first melting has been studied experimentally. ${ }^{23}$

To generate the a-Si interface with crystalline silicon (c-Si), we first equilibrated a $6 \times 6 \times 40$ diamond lattice with 11520 atoms for 11.04 ps at $T_{i o n}=1700^{\circ} \mathrm{K}$. Next, we create a liquid as before by heating half of the system at constant volume to $T_{i o n}=3000^{\circ} \mathrm{K}$ while keeping the other atoms fixed in place. Finally, the system is slowly annealed from $T_{i o n}=1700^{\circ} \mathrm{K}$ to $T_{i o n}=0$ at a rate of $3^{\circ} \mathrm{K} / p s$. During the annealing, the system dimensions in the plane of the interface are fixed, while the long dimension of the cell perpendicular to the interface is allowed to change and maintain a zero stress condition. At the end of the annealing, we obtain an a-Si region that is about $0.43 \mathrm{~nm}$ in thickness, and contains approximately 2300 atoms. We find most of the atoms in the a-Si to be four-fold coordinated $(\sim 88 \%)$, and the rest $(\sim 12 \%)$ are five-fold coordinated. The quality of the a-Si region is therefore comparable to the previous study using the same potential. ${ }^{13}$

We next annealed the a-Si/c-Si system using the empirical potential at three values of $k_{B} T_{e}$. The annealing was studied at constant temperature for a total simulation time of

TABLE II. Diffusion coefficients $D$ computed for the empirical potential for three different values of the electronic energy $k_{B} T_{e}$. In each case, $D$ is computed at the melting temperature $T_{m}$, also given in the table.

\begin{tabular}{lcc}
\hline \hline$k_{B} T_{e}$ & $T_{m}\left({ }^{\circ} \mathrm{K}\right)$ & $\mathrm{D}\left(\mathrm{cm}^{2} / \mathrm{s}\right) \times 10^{4}$ \\
\hline 0 & 1688 & 1.57 \\
0.5 & 1292 & 1.12 \\
1.25 & 594 & 0.21 \\
\hline \hline
\end{tabular}

1.66 ns. At $k_{B} T_{e}=0$, we find no growth of the crystal region for $T_{i o n}=300^{\circ} \mathrm{K}$ and $T_{i o n}=600^{\circ} \mathrm{K}$. However, for $T_{\text {ion }}=900^{\circ} \mathrm{K}$, we observe complete annealing of the a-Si region into crystalline silicon after $0.83 \mathrm{~ns}$, for a growth velocity of $2.62 \mathrm{~m} / \mathrm{s}$. This is somewhat surprising because experimental studies exhibit much slower annealing. For $k_{B} T_{e}=0.5 \mathrm{eV}$, we observe no growth of crystalline silicon up to $T_{i o n}=700^{\circ} \mathrm{K}$. However, it is possible that crystalline silicon will form closer to the melting point corresponding to $k_{B} T_{e}=0.5 \mathrm{eV}$, which is $T_{m}=1292^{\circ} \mathrm{K}$. For $k_{B} T_{e}=1.25 \mathrm{eV}$, we find no evidence of crystallization, and in fact observe an increase of about $0.5 \mathrm{~nm}$ in the a-Si region at $T_{i o n}=400^{\circ} \mathrm{K}$. Thus, it seems likely that for very high values of $k_{B} T_{e}$, crystalline silicon will not grow unless a liquid state is first reached.

\section{CONCLUSIONS}

In summary, we have developed an empirical potential for silicon that depends on $k_{B} T_{e}$. The parameters of the interaction potential are smooth functions of the electronic energy $k_{B} T_{e}$. The phonon spectra of the empirical potential follows that of the finite-temperature DFT calculations quite closely, indicating that the potential correctly captures the lattice instability that occurs under strong excitation. The dependence of the melting temperature $T_{m}$ has been determined, demonstrating that thermal melting might occur at a temperature far lower than what is expected in ordinary equilibrium melting. The kinetics of crystal growth for high $k_{B} T_{e}$ are much slower than under ordinary conditions. The selfdiffusion coefficient in the liquid state was also calculated, and found to be slower than for high $k_{B} T_{e}$ albeit only for much lower values of $T_{\text {ion }}$.

We did not succeed in directly annealing a-Si to form crystalline silicon without first forming a liquid state. However, it is possible that crystalline silicon can be formed at low values of $k_{B} T_{e}$ close to the melting temperature $T_{m}$, which itself depends strongly on $k_{B} T_{e}$. Another possibility is that liquid silicon does form in laser-annealing experiments, but at a temperature far below the ordinary melting temperature $T_{m}=1683^{\circ} \mathrm{K}$. However, very large values of $k_{B} T_{e}$ seem to make production of crystalline silicon more difficult, probably because crystalline silicon is much less stable under those conditions. More work, including realistic cooling of the system and exchange of energy between the electronic and ionic systems, is required to further elucidate annealing.

\section{ACKNOWLEDGMENTS}

This work was supported by a grant from the National Science Foundation (0809015). We also acknowledge support from a starter grant on the NSF teragrid, which was used for some of the calculations reported here.

\footnotetext{
${ }^{1}$ K. Sokolowski-Tinten and D. von der Linde, Phys. Rev. B 61, 2643 (2000).

${ }^{2}$ C. V. Shank, R. Yen, and C. Hirliman, Phys. Rev. Lett. 50, 454 (1983).

${ }^{3}$ A. M. Linderberg and J. Laarson, Science 308, 392 (2005).

${ }^{4}$ P. L. Silvestrelli, A. Alavi, M. Parrinello, and D. Frenkel, Phys. Rev. Lett. 77, 3149 (1996)
} 
${ }^{5}$ P. L. Silvestrelli, A. Alavi, M. Parrinello, and D. Frenkel, Phys. Rev. B 56, 3806 (1997).

${ }^{6}$ V. Recoules, J. Clerouin, G. Zerah, P. M. Anglade, and S. Mazevet, Phys. Rev. Lett. 96, 055503 (2006).

${ }^{7}$ A. Gambirasio, M. Bernasconi, and L. Colombo, Phys. Rev. B 61, 8233 (2000).

${ }^{8}$ P. Lorazo, L. J. Lewis, and M. Meunier, Phys. Rev. B 73, 134108 (2006).

${ }^{9}$ S. Khakshouri, D. Alfe, and D. M. Duffy, Phys. Rev. B 78, 224304 (2008).

${ }^{10}$ L. Shokeen and P. K. Schelling, Appl. Phys. Lett. 97, 151907 (2010).

${ }^{11}$ X. Gonze, J. M. Beuken, R. Caracas, F. Detraux, M. Fuchs, G. M. Rignanese, L. Sindic, M. Verstraete, G. Zerah, F. Jollet, M. Torrent, A. Roy, M. Mikami, P. Ghosez, J. Y. Raty, and D. C. Allan, Comp. Mat. Sci. 25, 478 (2002).

${ }^{12}$ T. Kumagai, S. Izumi, S. Hara, and S. Sakai, Comp. Mat. Sci. 39, 457 (2007).

${ }^{13}$ P. K. Schelling, Comp. Mat. Sci. 44, 274 (2008).
${ }^{14}$ M. Parrinello and A. Rahman, J. Appl. Phys. 52, 7182 (1981).

${ }^{15}$ U. Landman, W. D. Luedtke, R. N. Barnett, C. L. Cleveland, M. W. Ribarsky, E. Arnold, S. Ramesh, H. Baumgart, A. Martinez, and B. Khan, Phys. Rev. Lett. 56, 155 (1986).

${ }^{16}$ P. Keblinski, M. Z. Bazant, R. K. Dash, and M. M. Treacy, Phys. Rev. B 66, 064104 (2002).

${ }^{17}$ M. D. Kluge and J. R. Ray, Phys. Rev. B 39, 1738 (1989).

${ }^{18}$ G. J. Galvin, J. W. Mayer, and P. S. Peercy, Appl. Phys. Lett. 46, 644 (1985).

${ }^{19}$ J. Q. Broughton and X. P. Li, Phys. Rev. B 35, 9120 (1987).

${ }^{20}$ S. J. Cook and P. Clancy, Phys. Rev. B 47, 7686 (1993).

${ }^{21}$ F. H. Stillinger and T. A. Weber, Phys. Rev. B 31, 5262 (1985).

${ }^{22}$ C. P. Grigoropoulos, S. Moon, M. Lee, M. Hatano, and K. Suzuki, Appl. Phys. A 69, S295 (1999).

${ }^{23}$ T. Y. Choi, D. J. Hwang, and C. P. Grigoropoulos, Opt. Eng. 42, 3383 (2003). 